
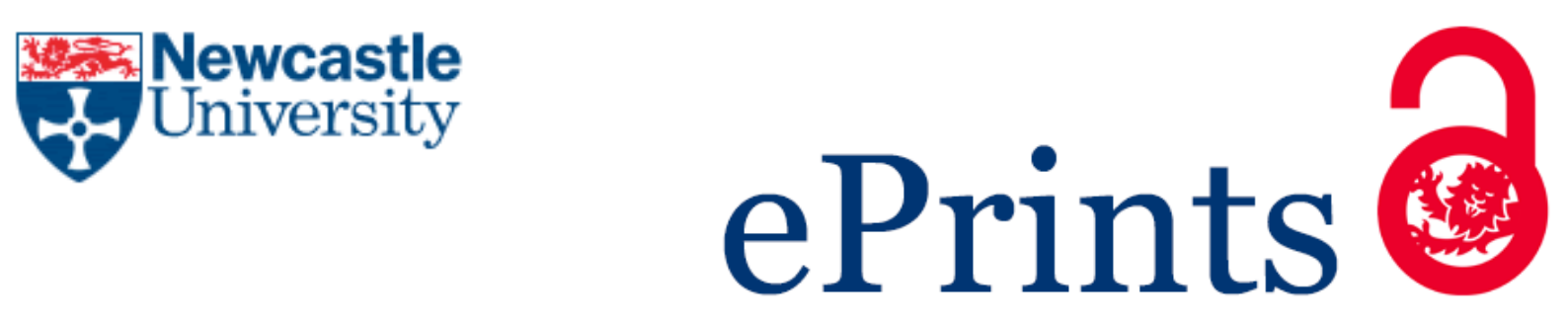

O'Brien KM, Timmons A, Butow P, Gooberman-Hill R, O'Sullivan E, Balfe M, Sharp L. Associations between neighbourhood support and financial burden with unmet needs of head and neck cancer survivors. Oral Oncology 2017, 65, 57-64.

\title{
Copyright:
}

(C) 2016. This manuscript version is made available under the CC-BY-NC-ND 4.0 license

DOI link to article:

http://dx.doi.org/10.1016/j.oraloncology.2016.12.019

Date deposited:

$15 / 06 / 2017$

Embargo release date:

28 December 2017

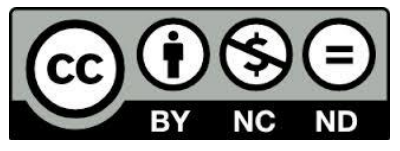

This work is licensed under a

Creative Commons Attribution-NonCommercial-NoDerivatives 4.0 International licence 


\section{Associations between neighbourhood support and financial burden with unmet needs in survivors of head}

and neck cancer.

\section{Abstract:}

Purpose

To assess the unmet needs of head and neck cancer survivors and investigate associated factors. In particular, to explore whether social support, (both close family/friends and neighbours) and financial burden are associated with unmet needs of head and neck cancer survivors.

\section{Methods}

This was a cross-sectional study of head and neck cancer survivors, with 583 respondents included in the analysis. Information was collected on unmet supportive care needs as measured by the Supportive Care Needs Survey (SCNS-SF34). Poisson regression with robust standard errors was used to examine factors associated with having one or more needs in each of the five domains (physical and daily living; psychological; sexuality; patient care and support; and health system and information).

\section{Results}

The mean age of respondents was 62.9 years (standard deviation 11.3 years) and one third of respondents were female. The top ten unmet needs was composed exclusively of items from the physical and psychological domains. Financial strain due to cancer and finding it difficult to obtain practical help from a neighbour were both associated with unmet needs in each of the five domains, in the adjusted analyses.

\section{Conclusion}

In each domain, a minority of respondents have unmet needs, with the commonest unmet needs in the psychological domain. Thus providing services to people with these needs should not place a large strain on healthcare providers. We suggest that studies such as ours, which identify risk factors for unmet needs, could be used to develop screening tools or aid in the targeting of support.

Keywords: head and neck cancer; unmet needs; social support; financial burden 


\section{Introduction}

In 2012, there were an estimated 686,000 new head and neck cancers diagnosed worldwide and 376,000 deaths from head and neck cancer [1], making it the seventh most common cancer worldwide both in terms of newly diagnosed cases and numbers of deaths [1]. Because of the close proximity of head and neck cancers to areas of anatomy involved in vital functions of daily living such as breathing, eating, drinking, and speaking, many survivors of head and neck cancer experience long-term side-effects of treatment which may be severe (NICE, 2004;[2]). The chronic nature of some of the side-effects experienced by some survivors mean that they can face a wide range of potentially overwhelming challenges as they adapt post-treatment and some individuals will need assistance to manage, or cope with, these challenges [3].

There is a paucity of information on supportive care needs for people diagnosed with head and neck cancer. A qualitative study carried out by Moore et al [7] explored supportive care needs of eight head and neck cancer survivors. They found that adjustment was adversely influenced by loss of access to the supportive hospital environment after treatment, which resulted in feelings of isolation post-treatment. The supportive care needs of 165 oral cancer patients from a hospital in northern Taiwan, were investigated by Chen et al [4]. The highest prevalence of unmet needs was in the area of health system and information. So et al [5] found that supportive care needs have a mediating effect on the relationship between characteristics of head and neck cancer survivors and their quality of life. Further work with a large representative sample is required to elucidate unmet needs focussing on survivors of head and neck cancer in the post-treatment phase using validated instruments.

An extensive literature has explored unmet needs in heterogeneous or other specific cancer types [6]-[8]. In their paper on supportive care needs of cancer patients in the immediate post treatment phase, Armes suggests that factors such as specific coping style and perceived social support may be influential in determining supportive care need [9]. McDowell et al [10] also found that a lower level of social support predicted unmet supportive care needs in the sexuality domain for patients/survivors with a variety of cancers and treatments. We postulated that a deficit in social support is associated with increased unmet needs in head and neck cancer survivors. Social support can come in the form of family, friends or neighbourhood support. Each of these offers different levels of support, and may be overlapping. In general, it is expected that deeper bonds are formed with family/friends than neighbours. Conversely neighbours may be more available to provide immediate practical support for everyday tasks. 
Associations between financial status and unmet needs have been reported ( [11] [18]) and Brooks et al [19] have demonstrated that financial hardship is common post-cancer diagnosis. This is likely to be particularly pertinent for head and neck cancer survivors, since the socio-economic distribution of this patient group suggests that many may be at risk of financial hardship. Post cancer financial hardship has been associated with a range of adverse outcomes including treatment non-adherence, poorer psychological wellbeing and lower health related quality of life ([14]-[17]).

Our aim, therefore, was to determine the prevalence of unmet needs amongst a large sample of medium- and long-term head and neck cancer survivors and the factors associated with survivors experiencing unmet needs. In particular, we were interested in investigating whether lower levels of social support and higher financial burden were associated with unmet needs after controlling for various clinical and demographic variables.

\section{Methods}

Setting

The study was conducted in Ireland, which has a mixed public-private health care system. Individuals are entitled to use the public health system; this includes hospital out-patient treatment and inpatient treatment in public hospitals. Public hospitals also offer private health care, and individuals may opt to be treated at private hospitals. Typically, those utilising private health care will have private health insurance. Possession of a medical card in Ireland entitles the holder to medical care free of charge within the public health system, including free primary general practitioner care. Medical cards are primarily awarded after means testing, though other factors like health status and age (over 70 years) are taken into account.

\section{Design and Participant Recruitment}

The study design was a cross-sectional, post-treatment survey of survivors of primary head and neck cancer receiving follow-up care at a range of hospitals throughout Ireland (14 hospitals which included all the major centres at which head and neck cancer is diagnosed and treated). A population based sample of head and neck cancer was identified from the Irish National Cancer Registry in April 2012. Within the definition of head and neck cancer, the following ICD 10 codes were included: cancers of the lip (COO), mouth (C01-C06), salivary glands (C07-C08), pharynx (C09-C14) and larynx (C32). Eligible individuals were invited to participate following the agreement of their main treating clinician. Exclusion criteria were as follows: the individual had 
died, was terminally ill, was unaware they had cancer or the treating clinician indicated that it would be inappropriate to contact them (e.g. they had dementia). Surveys with explanatory letters of invitation, and consent forms were sent to the 991 eligible individuals. Ethical approval was provided by the research ethics committee for each of the participating hospitals.

\section{Measures}

The Supportive Care Needs Survey, Short Form (SCNS-SF34) [18] was used to measure care needs of the respondents in "the last month". This instrument measures survivors' perceived need for support in five domains: physical and daily living; psychological; sexuality; patient care and support; and health system and information. In each domain we counted the number of people with one or more unmet needs for that domain.

The Oslo-3 social support scale was used to measure different aspects of social support [19]. The Oslo-3 scale contains three questions: How many people are you so close to that you can count on them if you have serious problems? (response options: 1-2, 3-5, more than 5); How much friendly interest do people show in what you are doing? (response options: a lot, some, uncertain, little, none); and How easy is it to get practical help from neighbours if you should need it? (response options: very easy, easy, possible, difficult, very difficult). A total score can be constructed or questions analysed separately. Cronbach's alpha for this data set was 0.62 , perhaps reflecting the multi-dimensional structure of the index. We therefore used the scores from the three questions separately in our analysis in order to explore which aspects of social support might be associated with unmet needs.

Financial burden was measured by including questions on household financial situation derived from previous work [14], [20]. Participants were asked about their household's ability to make ends meet immediately prior to their cancer diagnosis; henceforth financial stress at diagnosis (response options: 6-point Likert scale ranging from "very difficult" to "very easy"). They were also asked if their cancer diagnosis had made their household's ability to make ends meet more difficult; henceforth financial stress due to cancer (response options: 7-point Likert scale ranging from "much more difficult" to "much less difficult"). Finally, respondents were asked how concerned they were about their household's financial situation since the cancer diagnosis; henceforth known as 
financial strain due to cancer, (response options: 7-point Likert scale ranging from "much more concerned" to “much less concerned").

In addition, respondents were asked whether, at the time of diagnosis, they had private health insurance and whether they held a medical card. Information on a range of socio-demographic factors was available from the survey. Respondents were also asked if the head and neck cancer had returned or spread. Additional clinical information was obtained from the survivor's record with the National Cancer Registry. Stage was classified by TNM staging [21]. We combined stage I and II to form an early stage category and stages III and IV to form a late stage category.

\section{Statistical Analysis}

The ten most frequently endorsed unmet needs in the SCNS-SF34 were enumerated. In each of the five domains of the SCNS, we created a dichotomous outcome variable - at least one unmet need v. no unmet need. If an individual did not tick any boxes for the questions pertaining to a domain, their assignment for that domain was "unknown", and they were excluded from the analysis.

For each domain, we checked associations of the unmet needs with the main explanatory variables of interest (social support and financial burden) and the clinical and demographic variables using chi-squared tests. Pvalues less than or equal to 0.05 are considered statistically significant.

As the outcomes, unmet needs, were common (prevalence 14\%-33\%), instead of using logistic regression to estimate risk of unmet need for each domain, we modelled "at least one unmet need" using modified Poisson regression with robust variance estimation to evaluate risk ratios and associated $95 \%$ confidence intervals [22]. Variables were considered for inclusion in the multivariable model, if the associated p-value was at most 0.1 in the bivariate analysis. Then, a backward stepwise variable selection approach for choosing the multivariable model was used, removing variables in order of least significance until all retained variables achieved $\mathrm{p}<0.05$ in Wald tests. Care was taken to examine variables for multicollinearity and to assess their inclusion accordingly. Tests for interaction between time since diagnosis and the main co-variates social support and financial burden were examined when time since diagnosis was retained in the final model. 


\section{Results}

Respondents' characteristics

Completed surveys were received from 583 individuals (response rate 59\%). There were no statistically

significant differences between responders and non-responders in terms of gender, cancer site, stage at diagnosis and time since diagnosis (data not shown); however the non-responders were older $(\mathrm{p}=0.01)$. One third of respondents were female, with mean age 62.9 years (standard deviation 11.3 years), (Table 1).

Prevalence of reported unmet needs:

The top ten unmet needs was composed exclusively of items from the physical and psychological domains (Table 2). The percentage with at least one unmet need was highest in the psychological and physical domains $(32-33 \%)$, somewhat lower in the health system domain (21\%) and lowest in the sexuality and care and support domains $(14 \%)$.

Levels of social support and association with unmet needs:

For the 5 domains, table 3 shows associations between social support, financial burden and other demographic and clinical variables and having one or more unmet need. In terms of the number of people participants could count on if they had serious personal problems since their treatment finished, $21 \%$ reported that this was 1 or 2 , $35 \%$ reported that it was $3-5$ and $41 \%$ reported that it was more than 5 (Table 1). Having fewer people to rely on was significantly associated at the $5 \%$ level with having unmet needs in the physical/daily living, psychological, and health systems and information domains (Table 3).. Eighty eight percent of participants reported that since their treatment finished it was possible, easy or very easy to get practical help from a neighbour if they should need it and for $10 \%$ that this was difficult or very difficult. There was a strong significant association with having unmet needs in all domains and getting practical help from a neighbour.

\section{Financial burden and association with unmet needs:}

Almost a third of respondents had financial stress at diagnosis, while almost half had financial stress due to cancer (47\%). A similar proportion, 49\%, felt more concerned about household finances since their cancer diagnosis (financial strain due to cancer). Those with higher levels of financial stress and strain, demonstrated much higher levels of unmet needs across all domains, e.g. almost twice as many (48\% v. 25\%) of those with financial stress at diagnosis had physical unmet needs (Table 3). Financial strain due to cancer was strongly and 
significantly associated with all domains: in particular, half of those who were more concerned about the household financial situation post-diagnosis had unmet psychological unmet needs, compared to an unmet needs level of $17 \%$ in those who felt the same or were less concerned.

\section{Factors associated with unmet needs in multivariable analysis}

Two factors related to social support and financial circumstances were strongly associated with having one or more unmet needs in every domain after controlling for confounding variables; "obtaining practical help from a neighbour should you need it" and financial strain due to cancer (Table 4). Across all domains, those who found it difficult to obtain practical help from a neighbour had a 1.6-2.3-fold increased risk of unmet needs and those who were more concerned about their financial situation had 2.0-3.5 increased risk.

Other variables significantly associated with unmet needs in the physical needs domain were: financial stress at diagnosis, being female, having cancer recurrence, and having received chemotherapy or radiotherapy treatment.

In the psychological domain, the only additional variable which was significant in the multivariable analysis was time since diagnosis, with a lower risk of one or more unmet needs for those who were at least 5 years postdiagnosis. However, the interaction between time and financial strain is statistically significant, so that being more concerned about finances is associated with an increased risk of psychological unmet needs in the group with $<5$ years post diagnosis $(R R=1.95)$ and those with 5 or more years post-diagnosis $(R R=1.79)$ indicating that this risk factor does not diminish with time.

The risk of one or more unmet sexual needs was much reduced in the over 70s compared to those under $60 \mathrm{~s}$. The risk of at least one unmet need in the patient care and support domain was associated with having a medical card. In the health system and information domain, the risk of one or more unmet needs was also significantly associated with higher financial stress due to cancer and recurrence of head and neck cancer.

\section{Discussion}

Examining unmet needs in medium and long-term survivors of head and neck cancer can inform the tailoring of interventions or supports to the specific needs of these survivors, in order to enhance their quality of life [5]. Results from this analysis indicate that many (50\%) medium/long term survivors of head and neck cancer have 
no unmet needs at all - for many patients the health services are "doing well" for these patients. In each domain however, there is a minority who have unmet needs; $16 \%$ had at least one unmet need in one domains only, while $21 \%$ had unmet needs across three or more domains, with the commonest unmet needs in the psychological domain. This study therefore supports Armes [9] findings that a proportion of head and neck cancer survivors with unmet needs might benefit from the targeted application of psychosocial resources.

The question regarding "difficulty in getting practical help from a neighbour" was associated with unmet needs across all domains and remained a strong factor, even after adjusting for other variables in the multivariable analysis. While we postulated that less social support would be associated with unmet needs, we did not anticipate that the item "difficulty in getting practical help from a neighbour" from the Oslo-3 social support scale would be the strongest indicator in relation to unmet needs. Network members with stronger ties to, but not necessarily living close by, a person may provide more personal forms of support, e.g. emotional support or financial assistance, and this may sometimes be via phone, email or social media. In contrast, lack of neighbourhood contacts can lead to difficulties in obtaining practical help, creating more unmet needs and hence a negative cycle. Neighbour relations can be considered weak ties in a person's social network. Granovetter's [23] "strength of weak ties" argument suggests that weak ties provide enhanced connections to different social environments because they often connect dissimilar people, and these "bridging" ties can provide access to supportive information and services [24]. Neighbours can be a useful source of support for local level needs and provide a sense of being part of a community. Indirectly, ties which provide social support also help to give a sense of identification, self-worth and of social belonging [25] and neighbours generate a variation of this - a sense of belonging to a place [24]. Perhaps this spatial sense of belonging is a proxy for being able to navigate health and social systems to ensure that whatever needs a person has are attended to.

Balfe et al. have observed, in informal caregivers of the head and neck cancer survivors in this study, that there are also negative effects of social support, both in terms of accessing and receiving support (submitted to the European Journal of Cancer Care, 2016). It would be interesting to further investigate the interplay between social support, both instrumental and emotional, positive and negative, and unmet needs in head and neck cancer survivors. 
Financial strain due to cancer was associated with unmet needs in each of the domains. Financial stress and strain due to cancer, have been shown to be associated with adverse psychological outcomes in breast and prostate cancer patients in Ireland [14], [26] and thus the intersection with psychological unmet needs is not unexpected. In addition, health system inequalities may imply that some services may not be available due to their "pay at point of service" nature. The Irish health system has a complex structure and financing: both libertarian and egalitarian principles operate for different services and different individuals [27]. Many countries have mixed systems, but little is known about the financial impact of cancer in such settings. Timmons et al. observed that many health systems fail to offer citizens adequate financial protection because of insufficient financial risk pooling and prepayment mechanisms [34]; in general, in countries where out-of-pocket payments comprise a greater share of health funding, financial protection is worse.

It is noteworthy that all of the top ten unmet needs were from the physical and psychological domains. While the multivariable regression analysis suggests that those who have more than 5 years diagnosis have lower risk of unmet psychological needs, psychological unmet needs comprise seven out of the ten top needs in this sample and thus remain a priority area to be addressed. If survivors are not supported to regain as much function as possible, or to deal with the physical and psychological challenges they face, this can result in reduced social interactions and reduced psychosocial wellbeing [28], [29].

\section{Strengths and Limitations}

The survivors were sampled from a population-based sampling frame and enabled a large sample size for the study. Although the response rate was just under $60 \%$, the characteristics of responders and non-responders were largely similar. The only significant difference was that responders were younger. Younger age was associated with unmet sexuality needs but was not associated with the other domains, so we may have overestimated the prevalence of unmet sexuality needs. As this was a cross-sectional study the direction of effect cannot be determined. In particular, the two main variables associated with unmet needs, practical help from neighbour and financial burden, may be intertwined with unmet needs and so there may not be straightforward causal relationship. For example, having unmet needs might make one more concerned about one's financial situation. In addition, unmet needs may result in physical and psychological barriers that reduce one's ability to draw on social support, exasperating a negative feedback loop. 


\section{Implications}

The importance of supporting existing self-management processes as well as providing training and education to empower survivors of head and neck cancer and other chronic conditions has been highlighted [8], [38]. Our findings suggest that a needs assessment could be useful in developing a care plan for survivors. As many of the survivors do not have unmet needs, the effect on resources in the health sector should not be too onerous. Thus providing the services, to people with these needs (with particular focus on services related to physical and psychological needs) should not place a large strain on healthcare providers in Ireland. We suggest that studies such as ours, which look to identify risk factors for unmet needs, could be used to develop screening tools or aid in the targeting of support. 